\title{
On the applicability of the sieve bootstrap in time series panels
}

Citation for published version (APA):

Smeekes, S., \& Urbain, J. R. Y. J. (2011). On the applicability of the sieve bootstrap in time series panels. METEOR, Maastricht University School of Business and Economics. METEOR Research Memorandum No. 055 https://doi.org/10.26481/umamet.2011055

Document status and date:

Published: 01/01/2011

DOI:

10.26481/umamet.2011055

Document Version:

Publisher's PDF, also known as Version of record

\section{Please check the document version of this publication:}

- A submitted manuscript is the version of the article upon submission and before peer-review. There can be important differences between the submitted version and the official published version of record.

People interested in the research are advised to contact the author for the final version of the publication, or visit the DOI to the publisher's website.

- The final author version and the galley proof are versions of the publication after peer review.

- The final published version features the final layout of the paper including the volume, issue and page numbers.

Link to publication

\footnotetext{
General rights rights.

- You may freely distribute the URL identifying the publication in the public portal. please follow below link for the End User Agreement:

www.umlib.nl/taverne-license

Take down policy

If you believe that this document breaches copyright please contact us at:

repository@maastrichtuniversity.nl

providing details and we will investigate your claim.
}

Copyright and moral rights for the publications made accessible in the public portal are retained by the authors and/or other copyright owners and it is a condition of accessing publications that users recognise and abide by the legal requirements associated with these

- Users may download and print one copy of any publication from the public portal for the purpose of private study or research.

- You may not further distribute the material or use it for any profit-making activity or commercial gain

If the publication is distributed under the terms of Article $25 \mathrm{fa}$ of the Dutch Copyright Act, indicated by the "Taverne" license above, 


\section{Maastricht University}

Stephan Smeekes, Jean-Pierre Urbain

On the Applicability of the Sieve Bootstrap in Time Series Panels

$\mathrm{RM} / 11 / 055$

\section{METEOR}

Maastricht University School of Business and Economics

Maastricht Research School of Economics

of Technology and Organization

PO. Box 616

NL - 6200 MD Maastricht

The Netherlands 


\title{
On the Applicability of the Sieve Bootstrap in Time SERIEs PANels*
}

\author{
Stephan Smeekes Jean-Pierre Urbain \\ Department of Quantitative Economics \\ Maastricht University \\ December 15, 2011
}

\begin{abstract}
In this paper we investigate the validity of the univariate autoregressive sieve bootstrap applied to time series panels characterized by general forms of cross-sectional dependence, including but not restricted to cointegration. Using the final equations approach we show that while it is possible to write such a panel as a collection of infinite order autoregressive equations, the innovations of these equations are not vector white noise. This causes the univariate autoregressive sieve bootstrap to be invalid in such panels. We illustrate this result with a small numerical example using a simple bivariate system for which the sieve bootstrap is invalid, and show that the extent of the invalidity depends on the value of specific parameters. We also show that Monte Carlo simulations in small samples can be misleading about the validity of the univariate autoregressive sieve bootstrap. The results in this paper serve as a warning about the practical use of the autoregressive sieve bootstrap in panels where cross-sectional dependence of general from may be present.
\end{abstract}

Keywords: sieve bootstrap; panel data; cross-sectional dependence, final equations. JEL Classification: C23.

\section{Introduction}

In this paper we investigate the validity of the autoregressive (AR) sieve bootstrap in a panel data context. The sieve bootstrap is very popular in applied work as it is one of the better performing time series bootstrap methods, and moreover easy to implement. In particular

\footnotetext{
${ }^{*}$ Department of Quantitative Economics, Maastricht University, P.O. Box 616, 6200 MD Maastricht, The Netherlands. E-mail: S.Smeekes@maastrichtuniversity.nl, J.Urbain@maastrichtuniversity.nl. This research was supported financially by the Netherlands Organisation for Scientific Research (NWO). We are grateful to Alain Hecq, Marco Lippi and Franz Palm for helpful discussions. Special thanks go to Peter Boswijk for comments that drastically improved the paper. All remaining errors are our own.
} 
for the analysis of unit roots and cointegration, the sieve bootstrap appears to perform very well, see for example Chang, Park, and Song (2006) and Palm, Smeekes, and Urbain (2008).

Recently, people have also started to use the autoregressive sieve bootstrap in panel data with finite cross-sectional dimension. While a multivariate version of the sieve bootstrap exists based on the estimation of a vector autoregressive (VAR) model, this VAR sieve bootstrap becomes infeasible if the dimension of the system grows, and cannot be used in typical panel data applications where the number of cross-sectional units is too large, even in the finite $N$ case where it remains asymptotically valid. The VAR sieve bootstrap is therefore only of practical use in panels with a very small cross-sectional dimension where the estimation of a VAR model is justified. In applied work people therefore often use the univariate AR sieve bootstrap, with the modification that the residuals are resampled jointly across units to preserve the cross-sectional dependence. ${ }^{1}$ Examples for the analysis of unit roots and cointegration in panel data include Chang (2004), Cerrato and Sarantis (2007), Di Iorio and Fachin (2011), Hanck (2009), Smith, Leybourne, Kim, and Newbold (2004) and Westerlund and Edgerton (2007).

With the exception of Chang (2004) these papers do not provide theoretical results on the validity of the AR sieve bootstrap. Chang (2004) assumes that there is only contemporaneous dependence between units and shows that the AR sieve bootstrap is valid under those conditions. However, these conditions are likely to be violated in many empirical applications. It remains unknown if the AR sieve bootstrap is valid under more general conditions on the cross-sectional dependence. ${ }^{2}$

In this paper we therefore explore the properties of the AR sieve bootstrap in time series panels with more general forms of cross-sectional dependencies. Our main tool in this analysis is the final equations approach developed by Zellner and Palm (1974) and Palm (1977) among others, which shows that any VAR model can be written in final form as a system of ARMA equations for each individual unit. Starting from a general model that allows for various forms of cross-sectional dependencies, we use this approach as well as results of Kreiss, Paparoditis, and Politis (2011) to derive univariate AR representations for each unit that are needed in order to be able to apply the AR sieve bootstrap. However, as we will see, the innovations of these AR models are not vector white noise thus invalidating the use of the AR sieve bootstrap in more general models. Our focus is on unit root testing in panels, but our results carry through to other settings. Note that our analysis assumes that the cross-sectional dimension

\footnotetext{
${ }^{1}$ To distinguish between the multivariate sieve bootstrap where a VAR model is estimated and the univariate sieve bootstrap where individual autoregressive models are estimated, we refer to the former as VAR sieve bootstrap and to the latter as AR sieve bootstrap.

${ }^{2}$ The sieve bootstrap has been used in combination with some kind of estimation of a factor model to filter out dependence by Fuertes (2008), Pesaran, Smith, Yamagata, and Hvozdyk (2006), Trapani (2011) and Trapani and Urga (2010). While this is a perfectly sensible and valid approach, in this paper we only focus on the application of the sieve bootstrap directly to the data, hence where the sieve bootstrap is the only tool that takes into account the cross-sectional dependence. Trapani (2011) provides an extensive theoretical analysis of the sieve bootstrap (both of AR and VAR type) in a model where common factors are estimated.
} 
$N$, although potentially large, is finite. The same assumption is for example also made in Chang (2004).

We also illustrate our analysis with a numerical example of a model in which the AR sieve bootstrap is invalid but where observed size distortions are not large. This example serves as a possible explanation of the finding that the AR sieve bootstrap performs well in some simulation studies even though it is not valid.

The structure of the paper is as follows. In Section 2 we introduce a general sieve bootstrap algorithm used in panel data. The theoretical analysis is contained in Section 3. A small simulation study is considered in Section 4. Section 5 offers some conclusions. Proofs are contained in the Appendix.

Finally, a word on notation. $|z|$ applied to a (possibly complex) number denotes its absolute value, $\|A\|$ applied to any matrix $A$ denotes its Euclidean norm, and $\lfloor x\rfloor$ is the largest integer smaller than or equal to $x . W(r)$ denotes a standard $N$-dimensional Brownian motion. Weak convergence (convergence in distribution) is denoted by $\stackrel{d}{\rightarrow}$, and bootstrap weak convergence in probability is denoted by ${\stackrel{d^{*}}{\rightarrow}}_{p}$.

\section{Sieve bootstrap procedure}

Here we will describe a typical setup for an AR sieve bootstrap panel unit root test on a panel of data $y_{i, t}, i=1, \ldots, N, t=1, \ldots, T$.

1. For each $i=1, \ldots, N$, obtain $y_{i, t}^{d}=y_{i, t}-\hat{\beta}_{i}^{\prime} z_{t}$, where $z_{t}=1$ or $z_{t}=(1, t)^{\prime}$ and $\hat{\beta}_{i}$ is obtained for example through an OLS or GLS regression of $y_{i, t}$ on $z_{t}$.

2. For each $i=1, \ldots, N$, run an ADF regression with $q$ lags on $\Delta y_{i, t}$ to obtain residuals

$$
\hat{e}_{q, i, t}=\Delta y_{i, t}^{d}-\hat{\rho} y_{i, t-1}^{d}-\sum_{j=1}^{q} \hat{d}_{i, j} \Delta y_{i, t-j}^{d} .
$$

Recenter the residuals to obtain $\tilde{e}_{q, i, t}=\hat{e}_{q, i, t}-(T-q-1)^{-1} \sum_{t=q+2}^{T} \hat{e}_{q, i, t}$.

3. Resample with replacement from $\tilde{e}_{q, t}=\left(\tilde{e}_{q, 1, t}, \ldots, \tilde{e}_{q, N, t}\right)^{\prime}$ to obtain bootstrap errors $e_{t}^{*}=\left(e_{1, t}^{*}, \ldots, e_{N, t}^{*}\right)^{\prime}$.

4. For each $i=1, \ldots, N$, construct $u_{i, t}^{*}$ recursively as

$$
u_{i, t}^{*}=\sum_{j=1}^{q} \hat{d}_{i, j} u_{t-j}^{*}+e_{i, t}^{*},
$$

using the estimated parameters $\hat{d}_{i, j}$ from Step 2, and construct $y_{i, t}^{*}$ as $y_{i, t}^{*}=y_{i, t-1}^{*}+u_{i, t}^{*}$. 
5. Use the bootstrap sample $y_{t}^{*}=\left(y_{1, t}^{*}, \ldots, y_{N, t}^{*}\right)^{\prime}$ to calculate the desired panel unit root test statistic.

Lag lengths $q_{i}$ can be selected for each equation individually, for example by information criteria. We need to allow these lag length $q_{i}$ in the sieve bootstrap to go to infinity at a controlled rate.

Assumption 1. Let $q_{i} \rightarrow \infty$ and $q_{i}=o\left((T / \ln T)^{1 / 3}\right)$ as $T \rightarrow \infty$ for all $i=1, \ldots, N$.

\section{Invalidity of the sieve bootstrap in panel data}

In this section we will show theoretically that the AR sieve bootstrap is not valid in panel data with complex cross-dependencies. Let $y_{t}=\left(y_{1, t}, \ldots, y_{N, t}\right)^{\prime}$ and

$$
P(L) y_{t}=\left[(1-L) I_{N}-\alpha \beta^{\prime} L\right] y_{t}=\Psi(L) \varepsilon_{t}
$$

where $\alpha$ and $\beta$ are $N \times r$ matrices (with $r<N$ ). Furthermore we assume that

\section{Assumption 2.}

(i) $\varepsilon_{t}$ are i.i.d. with $\mathrm{E}\left(\varepsilon_{t}\right)=0, \mathrm{E}\left(\varepsilon_{t} \varepsilon_{t}^{\prime}\right)=\Sigma$ and $\mathrm{E}\left\|\varepsilon_{t}\right\|^{4}<\infty$.

(ii) $\operatorname{det}(\Psi(z)) \neq 0$ for all $z \in \mathbb{C}$ such that $|z| \leq 1$, and $\sum_{j=0}^{\infty} j\left\|\Psi_{j}\right\|<\infty$.

(iii) $\operatorname{det}\left(\alpha_{\perp}^{\prime} \beta_{\perp}\right) \neq 0$.

This DGP describes a multivariate $\mathrm{I}(1)$ process with possible cointegration. If $\alpha=0$, there is no cointegration, otherwise there are $r$ cointegrating relations. In the following we will not treat these two cases distinctively. We assume that $y_{0}=0$ and that there are no deterministic components present in the DGP. However, these assumptions are made purely for expositional simplicity and can be dispensed with without any difficulty.

By letting $\Phi(z)=\Psi(z)^{-1} P(z)$, we can derive the VAR representation

$$
\Phi(L) y_{t}=\varepsilon_{t}
$$

where $\Phi(1)=\Psi(1)^{-1} \alpha \beta^{\prime}$. We can further rewrite this to the VECM representation

$$
\Delta y_{t}=\Psi(1)^{-1} \alpha \beta^{\prime} y_{t-1}+\Phi^{*}(L) \Delta y_{t-1}+\varepsilon_{t},
$$

where the specific form for $\Phi^{*}(z)$ is given below equation (4) in Palm, Smeekes, and Urbain (2010). 
From the expression in (3) we can derive the common trends representation (see e.g. Johansen, 1995, Theorem 4.2)

$$
y_{t}=C \sum_{s=1}^{t} \varepsilon_{t}+C^{*}(L) \varepsilon_{t},
$$

where $C=\beta_{\perp}^{\prime}\left(\alpha_{\perp}^{\prime} \beta_{\perp}\right)^{-1} \alpha_{\perp} \Psi(1),{ }^{3} C^{*}(z)=\sum_{j=0}^{\infty} C_{j} z^{j}$ has all roots outside the unit circle and $\sum_{j=0}^{\infty} j\left\|C_{j}\right\|<\infty$ as a consequence of Assumption 2. It then follows that

$$
T^{-1 / 2} y_{\lfloor T r\rfloor} \stackrel{d}{\rightarrow} B(r),
$$

where $B(r)=C \Sigma^{1 / 2} W(r)$ is an $N$-dimensional Brownian motion with covariance matrix $\Omega=C \Sigma C^{\prime}$, which is equal to the long-run covariance matrix of $\Delta y_{t}$.

In order to be valid the AR sieve bootstrap should be able to replicate the invariance principle (5). Consequently, in order to be able to apply the AR sieve bootstrap, we need first to be able to write the DGP as a diagonal VAR process for $\Delta y_{t}$. To derive these univariate $\mathrm{AR}$ representations for $\Delta y_{i, t}, i=1, \ldots, N$, we use the final equations approach (see e.g. Zellner and Palm, 1974; Palm, 1977; Cubadda, Hecq, and Palm, 2009) and write the VAR form (2) as

$$
\operatorname{det}(\Phi(L)) y_{t}=\tilde{\Phi}(L) \varepsilon_{t}
$$

where $\tilde{\Phi}(z)$ is the adjoint matrix of $\Phi(z)$. Note that $\operatorname{det} \Phi(z)$ contains $N-r$ unit roots $(r=0$ if $\alpha=0)$. In order for $y_{t}$ to be I(1), $\tilde{\Phi}(z)$ must have $N-r-1$ unit roots. Hence, $N-r-1$ unit roots are common and cancel out. Hence, we may write $\operatorname{det}(\Phi(z))=a(z)(1-z)(1-z)^{N-r-1}$ and $\tilde{\Phi}(z)=B(z)(1-z)^{N-r-1}$, where $a(z)$ and $B(z)$ do not contain any factors $(1-z)$ (see Cubadda et al., 2009, for more details).

Let us further define $B_{i}(z)$ as the $i$-th row of $B(z)$, and $B_{i, j}(z)$ as the $(i, j)$-th element of the matrix $B(z)$. Then we may write for unit $i$ that

$$
a(L) \Delta y_{i, t}=B_{i}(L) \varepsilon_{t}=\sum_{j=1}^{N} B_{i, j}(L) \varepsilon_{i, t} .
$$

We still need to show that the right hand side of (6) can be written as a univariate invertible $\mathrm{MA}(\infty)$ process that can be inverted to obtain an autoregressive representation. Theorem 1 shows this is indeed possible.

Theorem 1. Let the DGP be given by (1) and let Assumption 2 hold. For each $i=1, \ldots, N$

\footnotetext{
${ }^{3}$ Note that $\alpha_{\perp}=I$ if $\alpha=0$ and hence $C=\Psi(1)$.
} 
we may write

$$
d_{i}(L) \Delta y_{i, t}=e_{i, t}
$$

where $d_{i}(z) \neq 0$ for $z \in \mathbb{C}$ such that $|z| \leq 1, \sum_{j=0}^{\infty} j\left|d_{i, j}\right|<\infty$ and $e_{i, t}$ is white noise with $\mathrm{E} e_{i, t}=0, \mathrm{E} e_{i, t}^{2}=\tilde{\sigma}_{i, i}$ and $\mathrm{E}\left(e_{i, t}^{4}\right)<\infty$.

Proof: see Appendix.

Note that it would be tempting to consider that the results in Theorem 1 provide a justification for the use of an univariate AR sieve bootstrap procedure in panel data. Indeed, all assumptions on the DGP needed in order to apply the sieve bootstrap (see e.g. Chang, 2004, Assumptions 1 and 2) are satisfied, with the exception that $e_{t}=\left(e_{1, t}, \ldots, e_{N, t}\right)^{\prime}$ is not a vector i.i.d. process. Unfortunately, this violation is serious enough to cause the sieve bootstrap to be theoretically invalid. There are two aspects of this violation that should be taken into account.

First, for each $i=1, \ldots, N, e_{i, t}$ is not i.i.d. but white noise. As explained in detail by Kreiss et al. (2011), this will only affect validity of the sieve bootstrap if the limiting distributions of the resulting test statistic depends on moments of $e_{i, t}$ higher than the second moment. The limit distributions of the unit root test statistics considered here are driven by the invariance principle (as in (5)) and only depend on the first two moments of the innovations. Therefore this does not lead to invalidity in this particular application, although it could do in others.

Second, while for each individual $i=1, \ldots, N, e_{i, t}$ is univariate white noise, $e_{t}$ is not vector white noise. This is a more serious problem that causes general invalidity of the AR sieve bootstrap, as the off-diagonal elements of the long-run covariance matrix of $e_{t}$ are not equal to those of the contemporaneous covariance matrix. To see why this is the case, let $D(z)=\operatorname{diag}\left(d_{1}(z), \ldots, d_{N}(z)\right)$, such that $e_{t}=D(L) y_{t}$. Note that we can write, using (4), that

$$
e_{t}=D(L) \Delta y_{t}=D(L) C \varepsilon_{t}+D(L) C^{*}(L) \Delta \varepsilon_{t}=\tilde{C}(L) \varepsilon_{t} .
$$

While $\mathrm{E} e_{i, t} e_{i, t-s}=0$ by construction for $s>0$, this will in general not be true for $\mathrm{E} e_{i, t} e_{j, t-s}$ if $i \neq j$. Define $\tilde{\Sigma}=\mathrm{E}\left(e_{t} e_{t}^{\prime}\right)$ and $\tilde{\Omega}=\lim _{T \rightarrow \infty} \mathrm{E}^{-1}\left(\sum_{t=1}^{T} e_{t}\right)\left(\sum_{t=1}^{T} e_{t}\right)^{\prime}$ and $\tilde{\sigma}_{i, j}\left(\tilde{\omega}_{i, j}\right)$ as the $(i, j)$-th element of $\tilde{\Sigma}(\tilde{\Omega})$. Then, while $\tilde{\omega}_{i, i}=\tilde{\sigma}_{i, i}$, in general we have that $\tilde{\omega}_{i, j} \neq \tilde{\sigma}_{i, j}$ for $i \neq j$.

Note that this result is irreducible, as we have found valid AR representations for each unit (making the univariate AR sieve bootstrap possible) that are derived from the fundamental ARMA models obtained through the final form. That is, there is no further transformation possible that will allow us to make the innovations vector white noise. Therefore, the AR sieve bootstrap is not valid when applied to a process generated by (1). 
The invalidity of the univariate AR sieve bootstrap is due to the fact that the bootstrap does not estimate, i.e. reproduce, the long-run covariance matrix of the data correctly. Using established results on the sieve bootstrap, see for example Chang (2004), Chang et al. (2006, Theorem 3.3) and Palm et al. (2010, Theorem 2), we can show that

$$
T^{-1 / 2} y_{\lfloor T r\rfloor}{\stackrel{d^{*}}{\rightarrow}}_{p} B^{*}(r),
$$

where $B^{*}(r)$ is an $N$-dimensional Brownian motion with covariance matrix $\Omega^{*}=D(1)^{-1} \tilde{\Sigma} D(1)^{-1 \prime}$, which will in general not be equal to $\Omega=D(1)^{-1} \tilde{\Omega} D(1)^{-1 \prime}$, the covariance matrix of $B(r)$ in (5).

Remark 1. Unfortunately there does not appear to be an intuitive way to link the DGP parameters to the parameters appearing in $\Omega$ and $\Omega^{*}$ (even for a bivariate $\operatorname{VAR}(1)$ model a complicated cumbersome expression arises that is difficult to interpret). It is therefore hard, if not impossible, to give simple conditions on the DGP considered here under which the AR sieve bootstrap is asymptotically valid. The only easily interpretable condition for sieve bootstrap validity is that $\alpha=0$ and $\Psi(z)$ diagonal, in which case the DGP reduces to the one used by Chang (2004). ${ }^{4}$

\section{Simulations}

To investigate the effect of the failure of the AR sieve bootstrap to capture the correct covariance matrix, will now analyze a very simple model by Monte Carlo simulation. We consider the following bivariate DGP:

$$
\left[\begin{array}{c}
\Delta y_{1, t} \\
\Delta y_{2, t}
\end{array}\right]=\left[\begin{array}{cc}
1 & \theta L \\
0 & 1
\end{array}\right]\left[\begin{array}{l}
\varepsilon_{1, t} \\
\varepsilon_{2, t}
\end{array}\right], \quad\left[\begin{array}{l}
\varepsilon_{1, t} \\
\varepsilon_{2, t}
\end{array}\right], \sim N\left(0,\left[\begin{array}{ll}
1 & 0 \\
0 & 1
\end{array}\right]\right) .
$$

Note that this process is actually in final form as $e_{1, t}=\Delta y_{1, t}$ and $e_{2, t}=\Delta y_{2, t}$ are white noise individually. Of course, if $\theta \neq 0$, they are not vector white noise. Although very simple, as shown in the previous section, this final form captures the relevant features of final forms arising from complex and rich dependence including cointegration between $y_{1, t}$ and $y_{2, t}$, because the diagonal AR dynamics are not of interest for AR sieve bootstrap validity, and the only important aspect is that the innovations are not vector white noise. Following Palm, Smeekes, and Urbain (2011), we will perform a group-mean (demeaned) Dickey-Fuller $t$-test, denoted $t_{g m}$, on these series using the sieve bootstrap.

\footnotetext{
${ }^{4}$ Trapani (2011) shows that, if the sieve bootstrap is applied in a panel model with a common factor structure that is estimated through principal components, the AR sieve bootstrap is valid for the long-run covariance matrix as long as the cross-sectional dependence between idiosyncratic components is "small", i.e. vanishing with $N$ (see his Theorem 3 for details). While this provides some guidance, such a statement only makes sense with increasing $N$ and is not applicable for our DGP.
} 


\subsection{Asymptotic Simulations}

We start with an asymptotic analysis of the AR sieve bootstrap. It follows directly (cf. Chang and Park, 2002; Chang, 2004) that

$$
\tau_{g m} \rightarrow^{d} \frac{1}{2} \sum_{i=1}^{2} \frac{B_{i}^{\mu}(1)^{2}-B_{i}^{\mu}(0)^{2}-\omega_{i, i}}{2 \sqrt{\omega_{i, i} \int_{0}^{1} B_{i}^{\mu}(r)^{2} d r}},
$$

where $B_{i}^{\mu}(r)=B_{i}(r)-\int_{0}^{1} B_{i}(r)$ and $B(r)=\left(B_{1}(r), B_{2}(r)\right)^{\prime}$ is a bivariate Brownian motion with covariance matrix given by

$$
\Omega=\left[\begin{array}{ll}
1 & \theta \\
0 & 1
\end{array}\right]\left[\begin{array}{ll}
1 & \theta \\
0 & 1
\end{array}\right]^{\prime}=\left[\begin{array}{cc}
1+\theta^{2} & \theta \\
\theta & 1
\end{array}\right],
$$

and $\omega_{i, i}$ is the $(i, i)$-th element of $\Omega$. On the other hand, for the sieve bootstrap statistic we have that

$$
\tau_{g m}^{*} \rightarrow d_{p}^{d^{*}} \frac{1}{2} \sum_{i=1}^{2} \frac{B_{i}^{* \mu}(1)^{2}-B_{i}^{* \mu}(0)^{2}-\omega_{i, i}^{*}}{2 \sqrt{\omega_{i, i}^{*} \int_{0}^{1} B_{i}^{* \mu}(r)^{2} d r}}
$$

where $B_{i}^{* \mu}(r)=B_{i}^{*}(r)-\int_{0}^{1} B_{i}^{*}(r)$ and $B^{*}(r)=\left(B_{1}^{*}(r), B_{2}^{*}(r)\right)^{\prime}$ is a bivariate Brownian motion with covariance matrix $\Omega=\left[\begin{array}{cc}1+\theta^{2} & 0 \\ 0 & 1\end{array}\right]$.

To investigate the asymptotic effect of the invalidity of the AR sieve bootstrap, we simulate the 0.05-quantiles of the distributions of $\tau_{g m}$ and $\tau_{g m}^{*}$ and the asymptotic rejection frequencies using a level of 0.05 . The asymptotic distributions were obtained by direct simulation of the relevant limiting representations, approximating the standard Brownian motion using i.i.d. $N(0,1)$ random variables, and with the integrals approximated by normalized sums of 2,000 steps. All simulations were performed using 500,000 Monte Carlo replications.

In Figure 1 we report the results for $\theta$ varying from -1 to 1 . Values for the quantiles are given on the right axis, while size is given on the left axis. While the quantiles are not very much different and follow the same pattern, they are clearly not identical. The invalidity of the sieve bootstrap is also confirmed by the reported asymptotic rejection frequencies. However, while they differ from the nominal level, the rejection frequencies are not that far away, and the asymptotic size distortion is not huge. ${ }^{5}$ Hence, in this particular model the effect of the off-diagonal parameter in the covariance matrix is fairly small, which may explain why the sieve bootstrap often still performs well in finite sample simulations.

\section{INSERT Figure 1 ABOUT HERE}

\footnotetext{
${ }^{5}$ Unreported results show that while for $|\theta|>1$ size distortions increase, size does not go above 0.12.
} 


\subsection{Finite sample simulations}

We next consider the same DGP for a finite sample Monte Carlo simulation. Sample sizes $T=50,100,250,500,1000,2000$ are considered. Results are based on 5000 simulations and 499 bootstrap replications. Next to the AR sieve bootstrap (SB) we also consider the i.i.d. bootstrap (IID) and the moving blocks bootstrap (MBB). As (8) is already in final form, the augmentation with lags in the AR sieve bootstrap is unnecessary. The i.i.d. bootstrap therefore plays the same role here as the AR sieve bootstrap; the only difference with the sieve bootstrap is that there will be no finite sample effect from the selection of the lag length. As such it basically provides a "clean" version of the sieve bootstrap here. The moving blocks bootstrap was shown to be valid in this context by Palm et al. (2011), and therefore provides a benchmark. ${ }^{6}$ The block length in the MBB is selected as $1.75 T^{1 / 3}$ as in Palm et al. (2011), while the lag length in the sieve bootstrap is selected by MAIC with an upper bound of $12(T / 100)^{1 / 4}$.

Figure 2 presents the results. For small to moderate $T$ it is difficult to see any difference between the valid moving blocks bootstrap and the invalid i.i.d. and AR sieve bootstrap. Only for a rather large $T$ does the bowl-shaped pattern found in Figure 1 become visible for the invalid bootstrap methods. Therefore, if one has no knowledge of Figure 1 and only considers sample sizes commonly considered in Monte Carlo studies, one might easily mistakenly believe the AR sieve bootstrap to be valid from these results.

\section{INSERT Figure 2 ABOUT HERE}

For the specific case considered here one might argue that while the AR sieve bootstrap is invalid, the asymptotic size distortion is so minor that it could be used in practice without problems. Even if one attaches value to this argument, it should be remembered that we only considered one very specific case here, especially as we took $N=2$. With a larger $N$ there is a wide range of parameters that can be selected and unreported simulations show that some combinations lead to major size distortions while others may lead to hardly any size distortions. Given the enormous array of possibilities it is not our goal here to investigate and report specific combinations. We have shown that the sieve bootstrap is invalid in this general type of models, and therefore the fact that parameters often chosen in simulation studies do not appear to lead to size distortions cannot be seen as validation for the AR sieve bootstrap.

\footnotetext{
${ }^{6}$ Clearly for this specific DGP we could have also used the VAR sieve bootstrap, given the small crosssectional dimension. We are however not interested in finding the best bootstrap method for this specific DGP. As mentioned before, the DGP is not of interest in itself, but serves as illustration for more general models, capturing the most important features. As the VAR sieve bootstrap is not applicable in more general models where $N$ is larger, we also do not consider it here.
} 


\section{Conclusion}

In this paper we have investigated the validity of the univariate autoregressive sieve bootstrap applied to a nonstationary multivariate system that allowed for general forms of cross-sectional dependence, including but not restricted to cointegration. While it was shown to be possible to write this system as a collection of infinite order univariate AR models, the innovations of these equations were shown not to be vector white noise, which causes the AR sieve bootstrap to be invalid in such a general system.

This result was illustrated with a numerical example using a simple bivariate system in which the AR sieve bootstrap was invalid. It was shown that the extent of invalidity depended on the value of the parameters. It was also found that in small or moderate samples the invalid AR sieve bootstrap was hard to distinguish from the valid moving blocks bootstrap, and only in large samples the asymptotic pattern could be recovered. This can explain the observation that the AR sieve bootstrap performs well in simulation studies performed in the literature.

The results of this paper have important implications for practitioners applying the autoregressive sieve bootstrap in a panel data setup, as it will typically be invalid for many settings it is applied to, unless cross-sectional dependence in the true DGP is only of a contemporaneous nature. Moreover, the bivariate example serves as a warning that relying upon Monte Carlo simulations to assess the validity of the AR sieve bootstrap can be very misleading. If one is not certain about the type of cross-sectional dependence present in the DGP, it is safer to use a different bootstrap method that is valid for a wider set of DGPs, such as the block bootstrap.

\section{References}

Brockwell, P. J. and R. A. Davis (1991). Time Series: Theory and Methods (2nd ed.). New York: Springer-Verlag.

Cerrato, M. and N. Sarantis (2007). A bootstrap panel unit root test under cross-sectional dependence, with an application to PPP. Computational Statistics 85 Data Analysis 51, 4028-4037.

Chang, Y. (2004). Bootstrap unit root tests in panels with cross-sectional dependency. Journal of Econometrics 120, 263-293.

Chang, Y. and J. Y. Park (2002). On the asymptotics of ADF tests for unit roots. Econometric Reviews 21, 431-447.

Chang, Y., J. Y. Park, and K. Song (2006). Bootstrapping cointegrating regressions. Journal of Econometrics 133, 703-739. 
Cubadda, G., A. Hecq, and F. C. Palm (2009). Studying co-movements in large multivariate data prior to multivariate modelling. Journal of Ecoometrics 148, 25-35.

Di Iorio, F. and S. Fachin (2011). A sieve bootstrap range test for poolability in dependent cointegrated panels. DSS Empirical Economics and Econometrics Working Papers Series 2011/2, Sapienza University of Rome.

Fuertes, A.-M. (2008). Sieve bootstrap t-tests on long-run average parameters. Computational Statistics \& Data Analysis 52, 3354-3370.

Fuller, W. A. (1996). Introduction to Statistical Time Series (2nd ed.). New York: Wiley.

Hanck, C. (2009). For which countries did PPP hold? A multiple testing approach. Empirical Economics 37, 93-103.

Johansen, S. (1995). Likelihood-Based Inference in Cointegrated Vector Autoregressive Models. Oxford: Oxford University Press.

Kreiss, J.-P., E. Paparoditis, and D. N. Politis (2011). On the range of validity of the autoregressive sieve bootstrap. Annals of Statistics 39, 2103-2130.

Palm, F. C. (1977). On univariate time series methods and simultaneous equation econometric models. Journal of Econometrics 5, 379-388.

Palm, F. C., S. Smeekes, and J.-P. Urbain (2008). Bootstrap unit root tests: comparison and extensions. Journal of Time Series Analysis 29, 371-401.

Palm, F. C., S. Smeekes, and J.-P. Urbain (2010). A sieve bootstrap test for cointegration in a conditional error correction model. Econometric Theory 26, 647-681.

Palm, F. C., S. Smeekes, and J.-P. Urbain (2011). Cross-sectional dependence robust block bootstrap panel unit root tests. Journal of Econometrics 163, 85-104.

Pesaran, M. H., R. P. Smith, T. Yamagata, and L. Hvozdyk (2006). Pairwise tests of purchasing power parity using aggregate and disaggregate price measures. Cambridge Working Papers in Economics 0634, University of Cambridge.

Smith, L. V., S. Leybourne, T.-H. Kim, and P. Newbold (2004). More powerful panel data unit root tests with an application to mean reversion in real exchange rates. Journal of Applied Econometrics 19, 147-170.

Teräsvirta, T. (1977). The invertibility of sums of discrete MA and ARMA processes. Scandinavian Journal of Statistics 4, 165-170.

Trapani, L. (2011). On bootstrapping panel factor series. CEA@Cass Working Paper Series WPCEA04-2011, Cass Business School. 
Trapani, L. and G. Urga (2010). Micro versus macro cointegration in heterogeneous panels. Journal of Econometrics 155, 1-18.

Westerlund, J. and D. L. Edgerton (2007). A panel bootstrap cointegration test. Economics Letters 97, 185190.

Zellner, A. and F. Palm (1974). Time series analysis and simultaneous equation econometric models. Journal of Econometrics 2, 17-54.

\section{A Appendix: Proof of Theorem 1}

Proof: Let $u_{i, t}=a(L) \Delta y_{i, t}$ as defined in (6). Then the first step is to show that

$$
u_{i, t}=c_{i}(L) e_{i, t}
$$

where $c_{i}(z) \neq 0$ for all $z \in \mathbb{C}$ such that $|z| \leq 1$ and $e_{i, t}$ is white noise. This is an extension of Theorem 1 of Teräsvirta (1977). As $u_{i, t}$ is a covariance stationary process, the Wold representation applies (see e.g. Brockwell and Davis, 1991, Section 5.7). Furthermore, as the Wold representation is fundamental, $c_{i}(z) \neq 0$ for all $z \in \mathbb{C}$ such that $|z|<1$. We still need to ensure that $c_{i}(z) \neq 0$ for all $z \in \mathbb{C}$ such that $|z|=1$, which is true if the spectral density of $u_{i, t}$ is strictly positive on $[-\pi, \pi]$. As shown in Teräsvirta (1977), the spectral density of $u_{i, t}$ is equal to

$$
f_{i}(\lambda)=\frac{1}{2 \pi} B_{i}\left(e^{-\mathrm{i} \lambda}\right) B_{i}\left(e^{\mathrm{i} \lambda}\right)^{\prime},
$$

which is zero at some point $\lambda_{i, 0} \in[-\pi, \pi]$ if only if all polynomials $B_{i, j}(z)$ for $j=1, \ldots, N$ have a common root on the unit circle at $e^{-\mathrm{i} \lambda_{i, 0}} .7$

As $B(z)$ does not contain any factors $(1-z)$, a common root cannot arise at frequency $\lambda=0$. Suppose there is a common root at another frequency $\lambda_{i, 0} \neq 0$. In that case we can factor out $\left(e^{-\mathrm{i} \lambda_{i, 0}}-z\right)$ from $B_{i}(z)$ and consequently we have $\operatorname{det}(B(z))=\left(e^{-\mathrm{i} \lambda_{i, 0}}-z\right) \tilde{B}(z)$ for some matrix polynomial $\tilde{B}(z)$. However,

$$
\operatorname{det}(B(z))=\frac{\operatorname{det}(\tilde{\Phi}(z))}{(1-z)^{N(N-r-1)}}=\frac{[\operatorname{det}(\Phi(z))]^{N-1}}{(1-z)^{N(N-r-1)}}=\frac{\left[(1-z)^{N-r} a(z)\right]^{N-1}}{(1-z)^{N(N-r-1)}}=(1-z)^{r} a(z)^{N-1}
$$

where $a(z)$ does not contain any unit roots as

$$
a(z)=\frac{\operatorname{det}(\Phi(z))}{(1-z)^{N-r}}=\frac{\operatorname{det}(P(z))}{\left(\operatorname{det}(\Psi(z))(1-z)^{N-r}\right)}=\frac{\operatorname{det}\left(P^{*}(z)\right)}{\operatorname{det}(\Psi(z))},
$$

\footnotetext{
${ }^{7}$ Teräsvirta (1977) considers MA models of finite order but his arguments are easily extended to infinite order MA models.
} 
which cannot contain any unit roots by the assumptions on $\Psi(z)$ and the fact that there are only $N-r$ unit roots in $\operatorname{det}(P(z))$ and consequently $\operatorname{det}\left(P^{*}(z)\right)=\operatorname{det}(P(z)) /(1-z)^{N-r}$ does not contain any unit roots.

As furthermore the unit roots $(1-z)^{r}$ cannot be the common roots as they occur at frequency zero, we can conclude that for each $i=1, \ldots, N$ there exist no common roots on the unit circle. Therefore the spectral density of $u_{i, t}$ is strictly positive for any $i=1, \ldots, N$, which proves (A.1).

We may then define $d_{i}(z)=\sum_{j=0}^{\infty} d_{i, j} z^{j}=a(z) c_{i}(z)^{-1}$, and write $d_{i}(L) \Delta y_{i, t}=e_{i, t}$ for $i=$ $1, \ldots, N$. Invertibility of $d_{i}(z)$ then immediately follows from (A.2) and the fact that $P(z)$ does not contain roots within the unit circle.

We next show that the summability condition holds. As $\Delta y_{t}=C \varepsilon_{t}+C^{*}(L) \Delta \varepsilon_{t}$ and $\sum_{j=0}^{\infty} j\left\|C_{j}\right\|<\infty$ from (4), it follows that $\sum_{h=-\infty}^{\infty} h\left|\mathrm{E} \Delta y_{i, t} \Delta y_{i, t+h}^{\prime}\right|<\infty$ for any $n=$ $1, \ldots, N$ (see e.g. Fuller, 1996, p. 367). It then follows from Lemma 2.1 of Kreiss et al. (2011) that $\sum_{j=0}^{\infty} j\left|d_{i, j}\right|<\infty$.

Finally, it follows from the Wold representation theorem that $e_{i, t}$ is white noise. From Assumption 2 and equation (4) one can conclude that $\mathrm{E}\left(\Delta y_{i, t}^{4}\right)<\infty$, and consequently that $\mathrm{E}\left(e_{i, t}^{4}\right)<\infty$. This concludes the proof. 


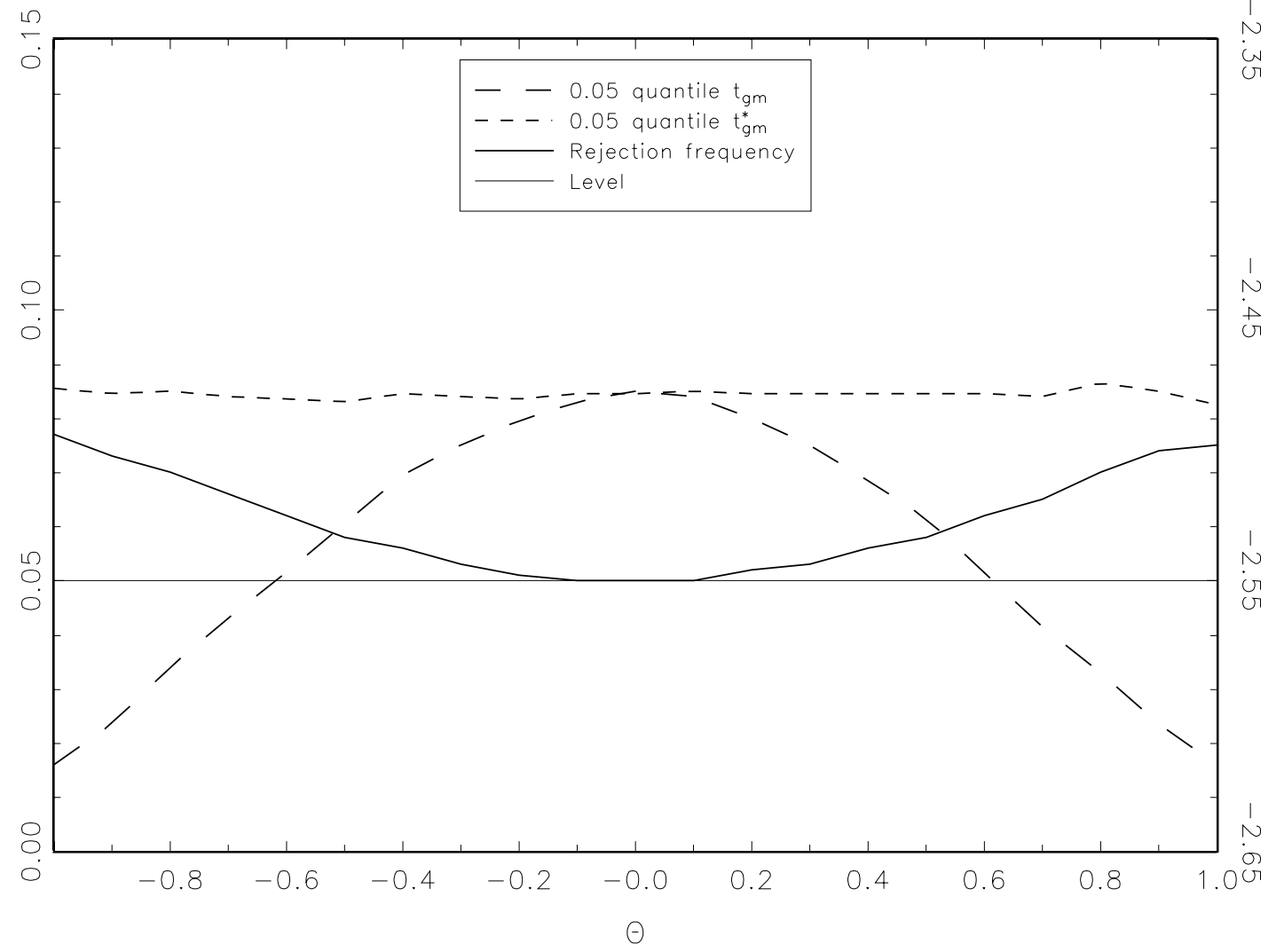

Figure 1: Asymptotic size and quantiles 


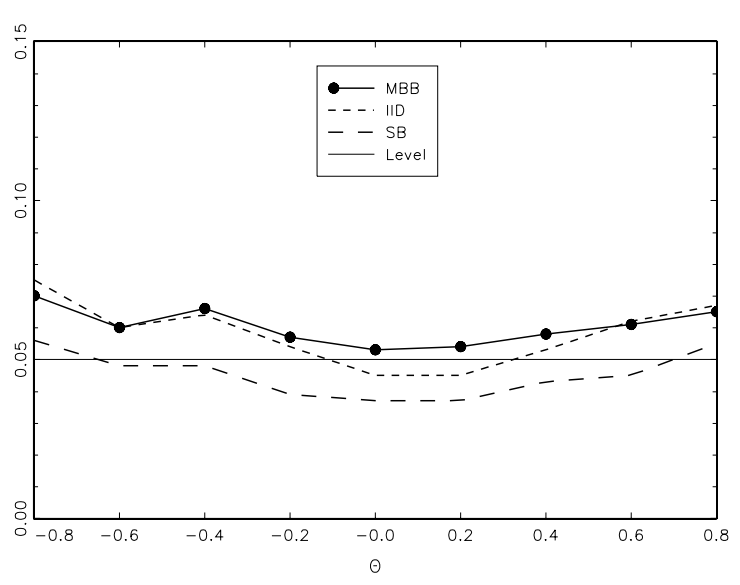

(a) $T=50$

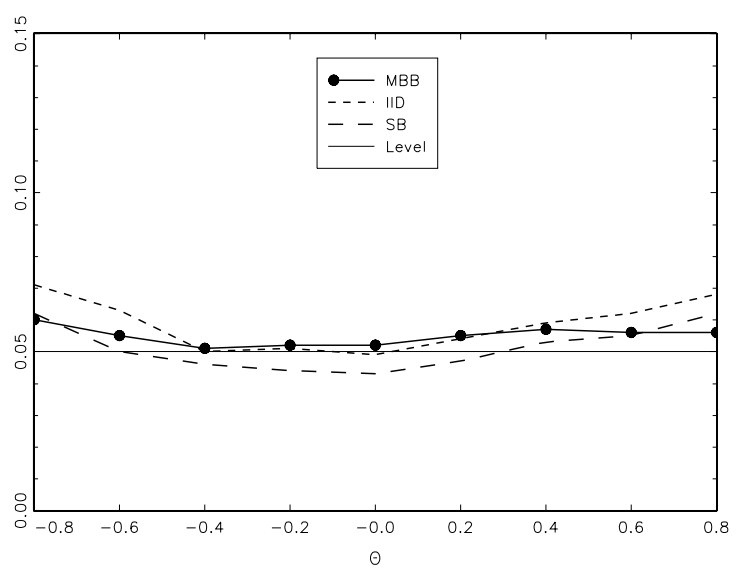

(c) $T=250$

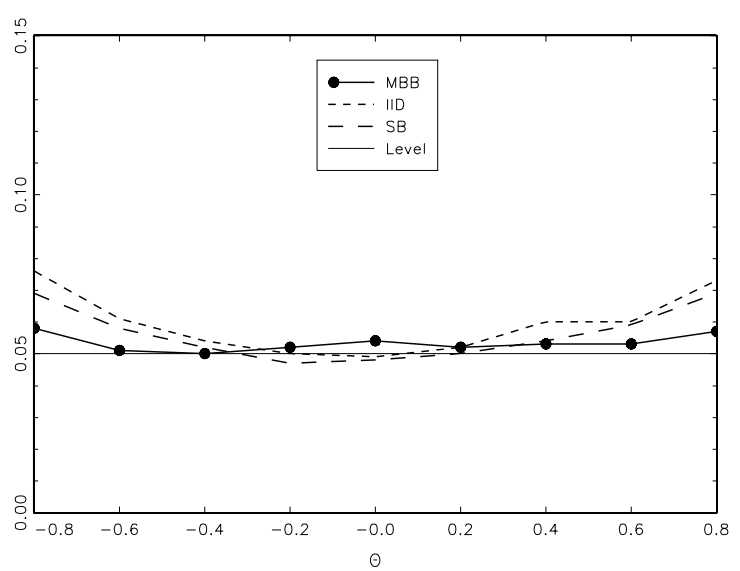

(e) $T=1000$

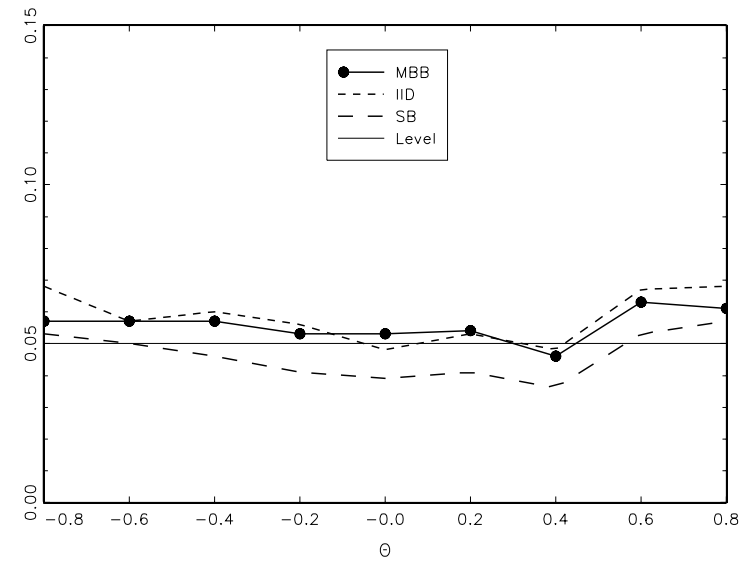

(b) $T=100$

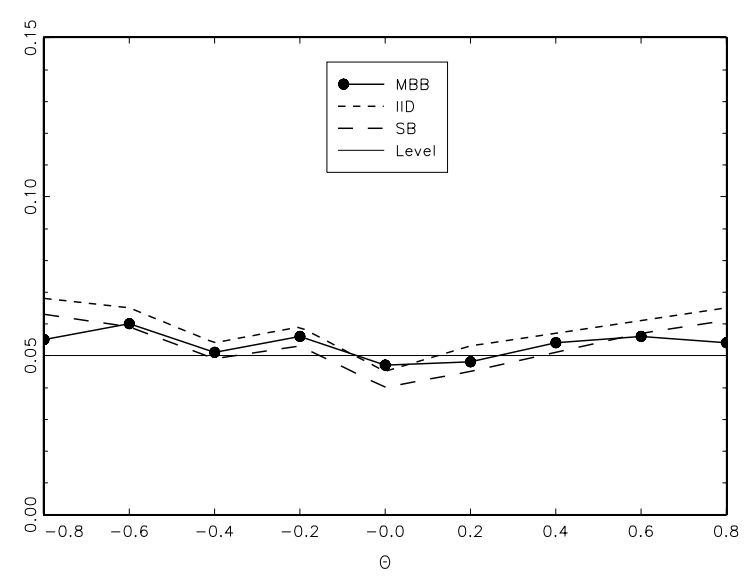

(d) $T=500$

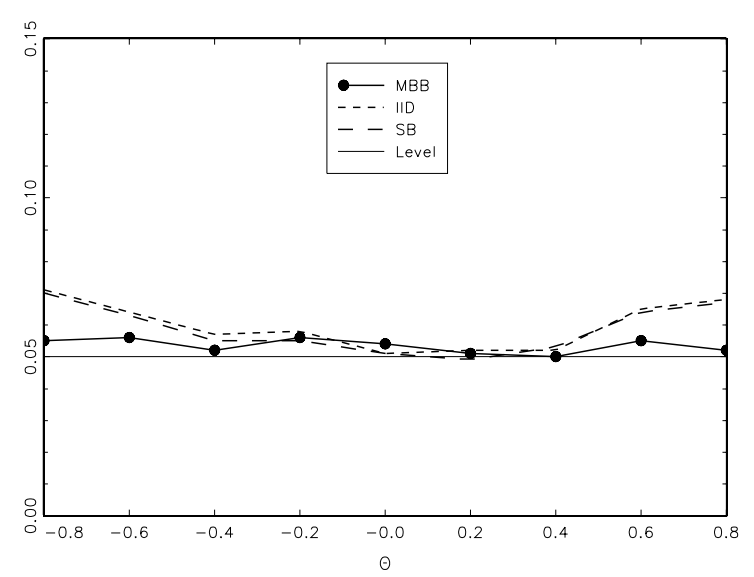

(f) $T=2000$

Figure 2: Size in finite samples 